\title{
URBAN CLASSIFICATION FROM AERIAL AND SATELLITE IMAGES
}

\author{
Iuliana Maria Pârvu ${ }^{\text {a, b* }}$, Iuliana Adriana Cuibac Picu ${ }^{\text {a, b* }}$, P.I. Dragomir ${ }^{c}$, \\ Daniela Poli ${ }^{d}$ \\ a, b* National Center of Cartography, 012101 / Doctoral School, Technical University of Civil Engineering of Bucharest, 020396, \\ Romania, e-mails: * iuliana.parvu@cngcft.ro / iuliana-maria.bina @ phd.utcb.ro, iuliana.cuibac@cngcft.ro / iuliana- \\ adriana.cuibac@phd.utcb.ro \\ ${ }^{\mathrm{c}}$ Faculty of Geodesy, Topography and Cadaster Department, Bucharest, Romania \\ ${ }^{\mathrm{d}}$ Vermessung AVT-ZT-GmbH, Imst, Austria, e-mail: d.poli@avt.at
}

Received: 30.05.2020 / Accepted: 10.07.2020/ Revised: 06.10.2020 / Available online: 15.12.020

DOI: 10.2478/jaes-2020-0024

KEY WORDS: photogrammetry, multispectral images, support-vector machine, maximum likelihood classifier.

\begin{abstract}
:
When talking about land cover, we need to find a proper way to extract information from aerial or satellite images. In the field of photogrammetry, aerial images are generally acquired by optical sensors that deliver images in four bands (red, green, blue and nearinfrared). Recent researches in this field demonstrated that for the image classification process is still place for improvement. From satellites are obtained multispectral images with more bands (e.g. Landsat $7 / 8$ has 36 spectral bands). This paper will present the differences between these two types of images and the classification results using support-vector machine and maximum likelihood classifier. For the aerial and the satellite images we used different sets of classification classes and the two methods mentioned above to highlight the importance of choosing the classes and the classification method.
\end{abstract}

\section{INTRODUCTION}

For urban and landscape analysis, photogrammetry and remote sensing allow to extract metric and thematic information of objects directly from aerial or satellite images, without being in contact with the objects themselves. Images can be acquired at different resolutions, in terms of geometry, spectrum, radiometry, and time, depending on the platform, the type of sensors and the application. In aerial photogrammetry largeformat multi-spectral cameras are used to acquire stereoscopic images with four bands (RGBI - Red, Green, Blue and Near Infrared) for mapping applications (i.e. 2D / 3D cartography. dense surface modelling, orthophoto production,). The scale of application is between scale 1:1.000 and 1:5.000, corresponding to a ground sampling distance (GSD) of the images between 2 and 30 centimetres. Photogrammetric aerial flights are executed at national scale on entire countries once every 2-3 years with the main purpose of generating true-colour or false-colour orthophotos, and update national spatial geodatabases. When combined with GIS (Geographic Information System) data, they are used for analysis, strategic planning and evaluation in urban planning and engineering. Hyperspectral cameras are also mounted on airplanes. They are medium-format cameras and images in larger spectral range with higher spectral resolution. For example, APEX camera (Vreys et al., 2016) acquires more than 300 bands in VNIR (Visible and Near InfraRed) range (380 - $870 \mathrm{~nm}$ ) and about 200 bands in SWIR (Short Wave InfraRed) range $(940-2500 \mathrm{~nm})$, while HySpex (HySpex, 2020) acquires about 200 and 300 bands in VNIR and SWIR ranges respectively.
Hyperspectral images are acquired in pushbroom mode, do not have stereo coverage for 3D analysis, and are used for thematic information extraction, land classification, environmental studies, and so on. Moving to satellite platforms, Earth observation satellites continuously acquire multi-spectral and hyperspectral imagery on any point of the Earth, with differences similar to those mentioned in the aerial case.

Multi-spectral optical sensor reach spatial resolution below 1 metre and stereo coverage since the beginning of 2000's for photogrammetric processing and $2 \mathrm{D} / 3 \mathrm{D}$ metric information extraction at a scale of 1:10.000 and lower. On a spectral point of view, four bands are acquired in the visible and infrared domains and spectral information, with four additional spectral bands centered in the RedEdge, yellow, coastal, and nearinfrared wavelengths, were introduced in 2015 with WorldView-2. The latest satellite of WorldView family, i.e. WorldView-3, launched in 2014, has brought the spatial and spectral resolution to extraordinary levels, by providing panchromatic images at a spatial resolution of $0.31 \mathrm{~m}$, eight multispectral bands at $1.24 \mathrm{~m}$, and eight additional Short Wave InfraRed (SWIR) bands at a spatial resolution of $3.7 \mathrm{~m}$ (DigitalGlobe, 2020). The images are available at a certain cost that depends on the area size, the age of the images, the order priority, the stereo availability and the type of licensing. Hyperspectral satellite images are widely available with a long historical archive. Similar to the airborne case, hyperspectral images do not have application in mapping, but in thematic information extraction, change detection and land cover classification. 
Indeed they have lower geometric resolution than multi-spectral ones, due to a different acquisition principle and technology, and acquire in a wider spectrum range with higher spectral resolution. In most cases the missions are financed by national space programs and images are available free of charge.

Satellite images are continuously acquired, with revisit time up to few days, in case of constellations of satellites, thus representing a fundamental source of information for change detection in landscape monitoring, disaster damage assessment, agricultural development and many other applications.

On the other hand aerial images are acquired on request; therefore they provide a straightforward depiction of the physical and cultural landscape of an area at a given time.

Aerial and satellite images are widely used for urban classification, and the choice of the bands and the data depends on the application and the data availability.

In this paper, we will focus on the classification of aerial images and satellite images on an urban environment, in order to evaluate the potential of each data and their applicability. As working environment, the software ENVI 5.3 (developed by Harris Corporation) was used. In Section 2 we will briefly report some historical notions about the development of aerial photogrammetry and related image processing. In Section 3 we will discuss the classification process and about two different classifiers: support vector machine (SVM) and maximum likelihood classifier (MLC). Section 4 is represented by the study case. The area selected is part of Săcueni City, Romania. We will see the results obtained using the classifiers and in Section 5 we will conclude our study.

\section{OVERVIEW OF AERIAL PHOTOGRAMMETRIC IMAGES AND SATELLITE IMAGES}

\subsection{Aerial photogrammetric images}

Photogrammetry can be defined in many ways, but the most straightforward is the following: "the science of obtaining reliable information about the properties of surfaces and objects without physical contact with the objects, and of measuring and interpreting this information" (Schenk, 2005). Based on the principles of trigonometry, photogrammetry relies on stereoscopic images taken from different locations. These images establish different "lines of sights" between each camera point and the object of interest. Through triangulating the intersections of these lines of sight, it is possible to determine the 3D location of the points of interest (Linder, 2006). Photogrammetry is classified, based on camera location during the acquisition of the data, in satellite, aerial and terrestrial photogrammetry. The platform used for acquiring aerial images has evolved from balloons and kites to airplanes, satellites and Unmanned Aircraft Systems (UAS). Aerial photogrammetry was first practiced by Gaspard-Félix Tournachon, in 1858, over the city of Paris, France. However, the images acquired then no longer exist and therefore the earliest aerial image (Fig. 1.) is the one taken by James Wallace Black and Samuel Archer King, in 1860, over Boston city, from a flight height of 630 meters. The most well-known applications for airborne photogrammetric products (Aerial Photography and Remote Sensing, 2014) are: land-use planning and mapping, geologic mapping, archaeology, and species habitat mapping.

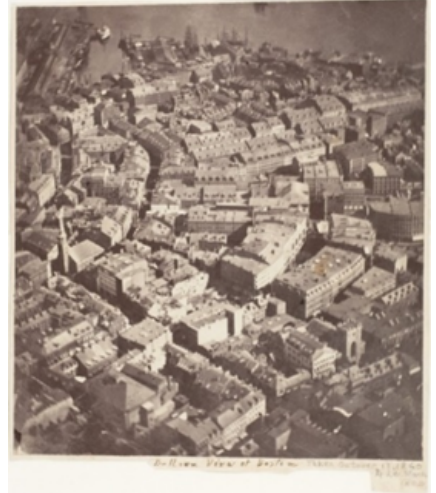

Figure 1. First aerial image over Boston city (Boston as the eagle and the wild goose see it, 2020)

In the time of analogue photogrammetry (1900-1960), it was discovered the first reliable black and white infrared sensor, which was used by the military sector. In the same period the color film was developed, which contains three emulsion layers sensitive to blue, green, and red. The computers were developed in the analytical photogrammetry period (1960-2000), and then the specialists converted the analogue images into digital images (made of pixels), by scanning process (Wolf et al., 2014). But the scanning involved loss of radiometric or tonal variation and spatial resolution (Leberl and Thurgood, 2004). The current step is digital photogrammetry (2000-present), which is characterized by the direct acquisition of digital images and their processing with computer software. Digital cameras use a charge-coupled device (CCD) or complementary metaloxide-semiconductor (CMOS) sensor to capture the image. The most common camera design is a rectangular matrix of millions of square sensor elements. Each of these pixels detects and records the amount of light received and the size of the pixel defines the spatial resolution. Aerial images for mapping applications are acquired in nadir (vertical) direction, with large format matrix cameras, like the UltraCam Eagle Mark 3 (Vexcel) and the Leica DMC II (Leica). In the year 1860 the first oblique airborne image was acquired (Fig. 1.), but this was only the beginning. Recently photogrammetric multi-sensors oblique aerial cameras have been introduced to the market of multi-view image acquisition and mapping in urban environments. The actual oblique camera systems come in a variety of configurations. Review and state-of-the-art of oblique systems are reported in (Remondino et al., 2014), (Karbo and Schroth, 2009), (Lemmens, 2011) and (Petrie, 2009). Nowadays, the most known oblique airborne systems are UltraCam Osprey (Vexcel), Leica RCD30 Oblique (Leica), IGI Quattro DigiCAM Oblique (IGI).

The standard workflow for orthophoto production consists of flight and field measurement planning, aerial data acquisition, field survey of ground control points and check points, trajectory estimation, radiometric image pre-processing, aerial triangulation for the calculation of the image orientation, image orthorectification on a suitable Digital Terrain Model (DTM). Additionally, if the overlap is sufficiently big, digital surface model (DSM) can be automatically generated with dense image matching algorithms.

\subsection{Satellite images}

Remote Sensing refers to the branch of science which derives information about objects from measurements made from a 
distance (Navalgund, 2001). The first images from space were taken on the sub-orbital V-2 rocket flight, launched by the United States, in the year 1946 (Fig. 2).

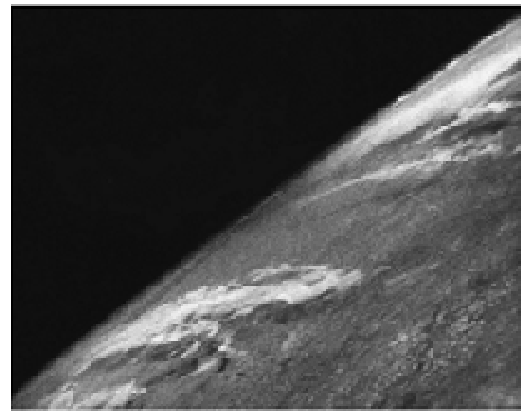

Figure 2. Image of Earth from Space (First photo from space, 2020)

The concept of satellite imagery refers to digitally transmitted images taken by artificial satellites orbiting the Earth. The first successful weather satellite, Tiros-1, was launched by the National Aeronautics and Space Administration (NASA). It transmitted infrared images of Earth's cloud cover and was able to detect and chart hurricanes. In 1972, NASA launched the Earth Resources Technology Satellite; this was the start of the longest-running program of satellite imagery of the Earth, later renamed Landsat.

Landsat instruments acquire millions of images that are used to evaluate natural and human changes of the Earth. Landsat 8 was launched in 2013 and has two sensors on-board: Operational Land Imagery (OLI) and Thermal Infrared Sensor (TIRS). Landsat 8 measures in 11 ranges of frequencies along the electromagnetic spectrum and the products have three different spatial resolutions: 15 meters (panchromatic), 30 meters (visible, near-infrared and short-wavelength infrared) and $100 \mathrm{~m}$ (thermal) (Landsat Program, 2020).

The European Space Agency (ESA) developed a new family of satellites in the Copernicus program. These missions carry a range of technologies (radar and multispectral imaging) for monitoring land, ocean, and atmosphere. Sentinel-1 is a radar imaging mission for land and ocean services. Sentinel-2 is a multispectral imaging mission for land monitoring to provide, for example, imagery of vegetation, soil and water cover, inland waterways and coastal areas.

Sentinel-3 is a multi-instrument mission to measure sea-surface topography, sea- and land-surface temperature, ocean color and land color with high-end accuracy and reliability. Sentinel-5P provides timely data on a multitude of trace gases and aerosols affecting air quality and climate. Sentinel-4 and Sentinel-5 are payload devoted to atmospheric monitoring. Finally, Sentinel-6 carries a radar altimeter to measure global sea-surface height, primarily for operational oceanography and climate studies (Sentinel 2, 2012). Nowadays various space agencies and satellite product providers have adopted a free and unrestricted data access policy for satellite data. We can recall here, the Copernicus program (ESA), over five million Landsat images from 1972 onwards (U.S. Geological Survey), the 30 meters Digital Elevation Models (Japan Aerospace Exploration Agency and NASA), etc.
When talking about digital images characteristics, four types of resolutions are important. Spatial resolution represents the size of the section of the Earth's surface which can be depicted in one pixel and radiometric resolution indicates the ability of the sensor to distinguish between grey-scale values and is measured in bits.

Dealing with thematic applications, spectral resolution indicates how well a spectral-digital sensor can distinguish between the different spectral ranges of the electromagnetic spectrum and the temporal resolution indicates the distance in time between two successive image acquisitions of the same area.

To view and analyse satellite images, a pre-processing is needed to remove the atmospheric effect (Fig. 3), correct the radiometry and improve the geometric properties.

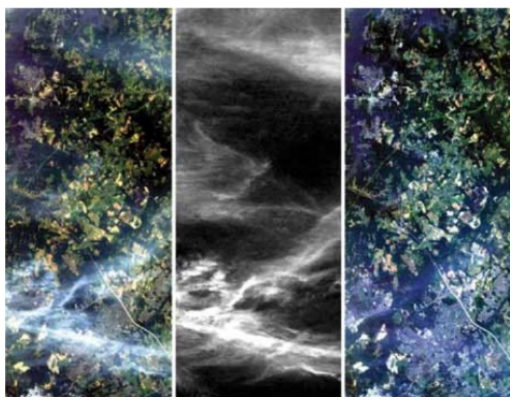

Figure 3. Example of simulated Sentinel-2 data before and after atmospheric correction (Sentinel 2, 2012)

\section{OVERVIEW ON CLASSIFICATION PROCESSES}

One of the main application of aerial and satellite images is the classification for land cover information extraction (Kramer, 2002), (Foody and Mathur, 2004).

Image classification is the process of grouping pixels into several classes of land use or land cover, based on statistical decision rules in the multispectral domain or logical decision rules in the spatial domain. The methods and the algorithms used in the classification process are called image classifiers (Kamavisdar et al., 2013). The classification process consists of the following steps:

- Pre-processing: analysing the input data by interpreting and understanding it and defining the number of classes for classification;

- Training: creating the samples for each class used for the training and the validation step.

- Applying the image classifier: running the desired classification methods.

- Post classification: assessment of accuracy (Foody, 2002).

The classification process (Fig. 4.) is categorized, in supervised and unsupervised classification, based on the use or not of training samples. In the next paragraphs the most common classifiers are presented. 


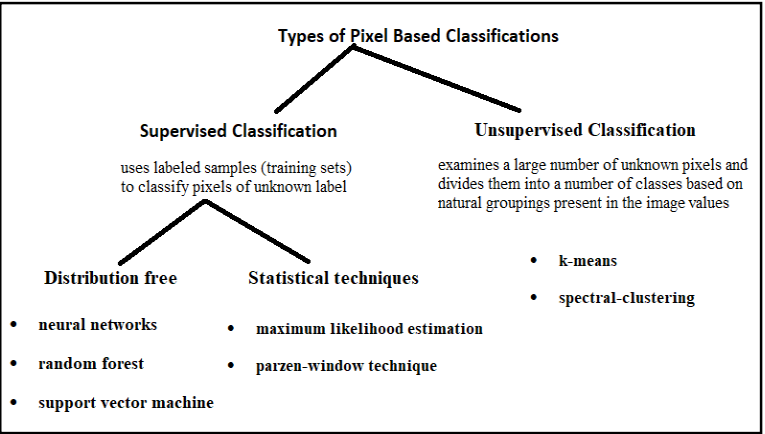

Figure 4. Types of image pixel-based classification

\subsection{SVM}

Support-Vector Machine (SVM) algorithm has its roots in Statistical Learning Theory (Vapnik, 1995). SVM is a supervised learning model with associated learning algorithms that analyse the data used for classification. In the beginning, given a set of training samples, each marked as belonging to one or the other of two classes, SVM training algorithm built a model that assigns a label to every pixel, as belonging to one of the classes, making it a non-probabilistic binary linear classifier.

The characteristic that defines the SVM classifier is that its purpose is to find a hyperplane (Fig. 5 and 6.) in an $\mathrm{N}$ dimensional space ( $\mathrm{N}$ is the number of features), that distinctly classifies the data points (Gandhi, 2018).

Hyperplanes are decision boundaries that help to classify the data points, that is, data points falling on either side of the hyperplane are attributed to different classes. Also, the dimension of the hyperplane depends upon the number of features. If the number of classes is two, then the hyperplane is just a line, if the number of input features is three, then the hyperplane becomes a plane. It becomes difficult to imagine when the number of features exceeds 3 .
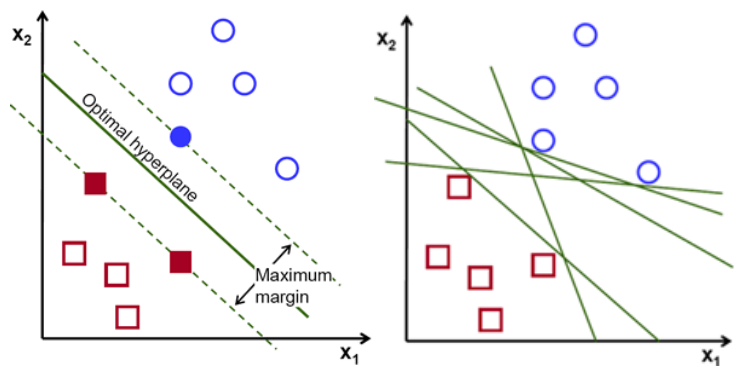

Figure 5. Hyperplanes in 2D space (Gandhi, 2018)
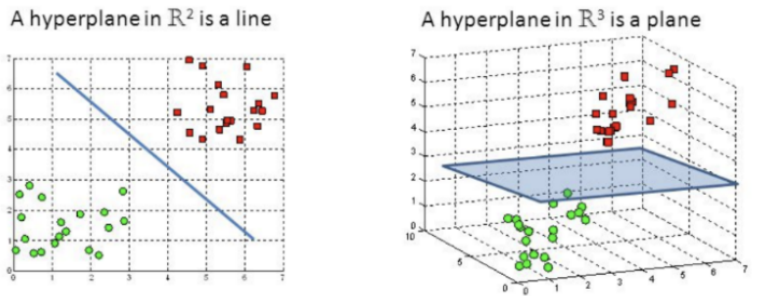

Figure 6. 2D and 3D hyperplanes (Gandhi, 2018)

\subsection{MLC}

Maximum Likelihood Classifier - MLC (Duda and Hart, 1973), also called Discriminant Analysis, is the most popular statistical method of classifying images. According to this approach, a pixel with the maximum likelihood is classified into the corresponding class. The steps involved in MLC process are the following:

- computing the parameters that represent the data (standard deviation, sigma, mean, etc.), for each class;

- computing the probability density of every feature vector to each class, based on the parameters calculated in the prior step;

- $\quad$ finding the maximum of all the computed probability densities for each class. The class with the parameters that give the maximum probability is the predicted class of the data point.

A simplified version of this classifier is given in equation (1).

$$
p\left(x \mid \omega_{j}\right)=\frac{1}{\sigma_{j} \sqrt{2 \pi}} e^{-\frac{1}{2}\left(\frac{x-\mu_{j}}{\sigma_{j}}\right)^{2}}
$$

where:

$$
\begin{aligned}
& \omega_{j}=\text { set of parameters for each class } \mathrm{j} ; \\
& \sigma_{j}=\text { standard deviation for the class } \mathrm{j} ; \\
& \mu_{j}=\text { mean for the class } \mathrm{j} ; \\
& x=\text { feature vector. }
\end{aligned}
$$

The standard deviation and the mean of every class are used as parameters, but with the MLC model other parameters can be used as well. In equation (1) the correlation between features is neglected. The full version of MLC, implemented in remote sensing software, is described by equation (2).

$$
d_{k}(g)=-\log \left|\Sigma_{k}\right|-\left(g-\mu_{k}\right)^{T} \Sigma_{k}^{-1}\left(g-\mu_{k}\right)
$$

where:

$$
\begin{gathered}
\Sigma_{k}=\text { sum of all standard deviations } ; \\
\left(g-\mu_{k}\right)^{T} \Sigma_{k}^{-1}\left(g-\mu_{k}\right)=\text { Mahalanobis Distance. }
\end{gathered}
$$

An advantage of using the Mahalanobis distance is that it takes into consideration the correlation between classes. When using MLC a sufficiently large ground truth sampled data is required to allow the estimation of the unknown parameters.

\subsection{Accuracy assessment}

Accuracy assessment is an important part of any classification process. In this step, the classified image is compared to a data source that is considered the ground truth data. Ground truth can be collected in the field or can be derived from interpreting high-resolution imagery, from existing classified imagery, or from existing GIS data layers.

The relationship between the known reference data (ground truth) and the corresponding results of the classification procedure is stored in the confusion matrix. The accuracy indicators are then computed based on this matrix. 
Overall Accuracy describes the percentage of correctly classified pixels from the total number of reference pixels.

The Kappa Coefficient is generated from a statistical test to evaluate the accuracy of the classification. Kappa evaluates how well the classification performed as compared to just randomly assigning classes. The values can range from -1 to 1 . A value of 0 indicates that the classification is no better than a random one. A value close to 1 indicates that the classification is better than random.

Errors of omission refer to reference samples that were left out (or omitted) from the correct class in the classified map. To compute the omission for every class, the incorrect classifications are added and divided by the total number of reference samples.

Errors of commission are calculated by reviewing the classified samples for incorrect classifications. This is done by adding together the incorrect classifications and dividing them by the total number of classified samples for each class.

Producer's Accuracy is the map accuracy of the mapmaker (the producer) and represents how often real features on the ground are correctly shown on the classified map. It is calculated as the number of correctly classified pixels divided by the total number of reference pixels for that class.

The User's Accuracy is the accuracy for a map user and is referred to as reliability. It indicates how often the class on the map will be present on the ground. The User's Accuracy is calculated by dividing the total number of correctly classified pixels for a class by the number of pixels that were classified in that class.

\section{CASE STUDY}

The area chosen for the tests is located in Săcueni City in Bihor County, Romania (Fig. 7).

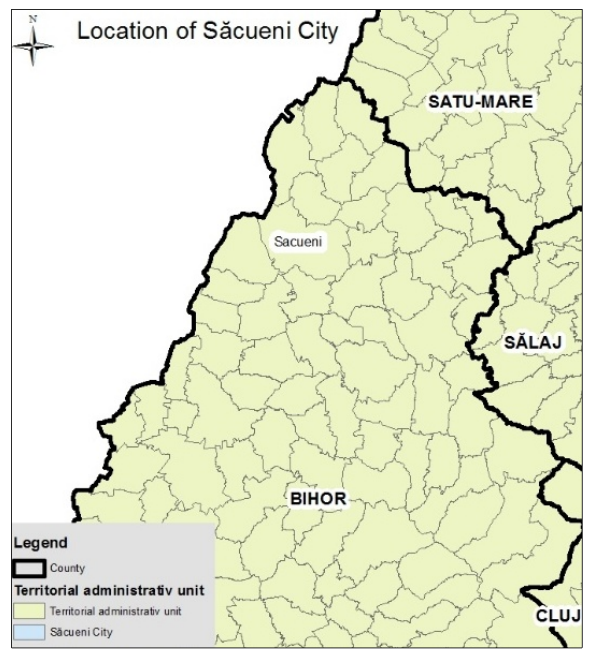

Figure 7. Săcueni City, Romania

The study case analyses the results achieved in image classification using two different sources of data, from airborne and satellite platforms. In the next paragraphs the input data, the corresponding products obtained, the results of the classification using two different classifiers and the comparison of the results are presented.

\subsection{Classification of orthophotos}

The first type of image data was acquired during a photogrammetric aerial flight within the project LAKI II, that started in 2017 (Fig. 8.) and covers an area of $50.000 \mathrm{~km} 2$ in the western and central part of Romania. For the acquisition of aerial images the UltraCam Eagle Prime camera was used, mounted on a Twin Commander 690A airplane. For image classification, a true-color (RGB) orthophoto with ground resolution 20 centimeters and a planimetric accuracy of 30 centimeters was used (Fig. 9).

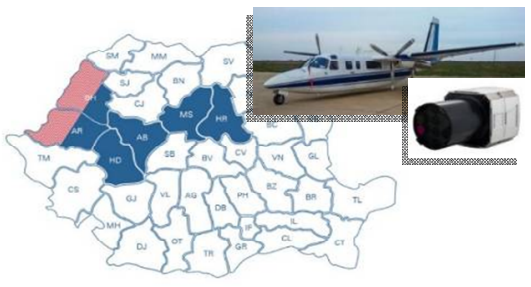

Figure 8. Area for LAKI II Project and the photogrammetric camera used for data acquisition

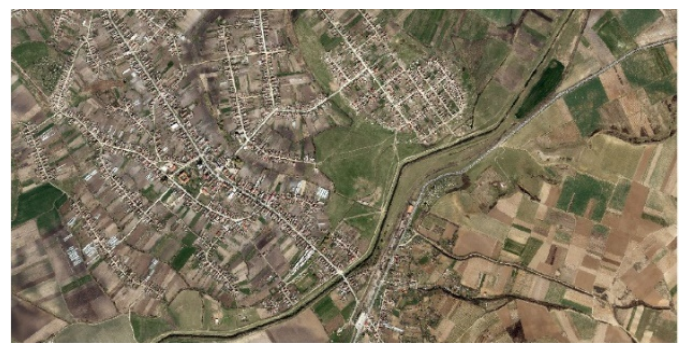

Figure 9. Aerial orthophoto used for image classification

The first step in the classification process was image interpretation in order to configure the classes to be extracted. Based on the land use and land cover characteristics, the following classes were selected: BUILDINGS, ROADS, GRASS, BARE-EARTH and WATER.

In ENVI 5.3 software, the samples were digitized for training and reference (Table 1). These samples must be uniformly distributed across the image (as far as possible) and must contain the most representative areas for the corresponding class (Fig. 10). As classifiers, both SVM and MLC.

\begin{tabular}{|c|c|c|c|c|}
\hline \multirow{2}{*}{ Classes } & \multicolumn{2}{|c|}{ Training dataset } & \multicolumn{2}{c|}{ Reference dataset } \\
\cline { 2 - 5 } & $\begin{array}{c}\text { No. } \\
\text { polygons }\end{array}$ & $\begin{array}{c}\text { No. } \\
\text { pixels }\end{array}$ & $\begin{array}{c}\text { No. } \\
\text { polygons }\end{array}$ & $\begin{array}{c}\text { No. } \\
\text { pixels }\end{array}$ \\
\hline BUILDINGS & 20 & 11456 & 15 & 10319 \\
\hline ROADS & 14 & 17380 & 12 & 17239 \\
\hline GRASS & 15 & 39574 & 15 & 41472 \\
\hline BARE-EARTH & 15 & 50561 & 15 & 58401 \\
\hline WATER & 5 & 10590 & 5 & 13099 \\
\hline
\end{tabular}

Table 1. Number of samples for training and reference dataset 


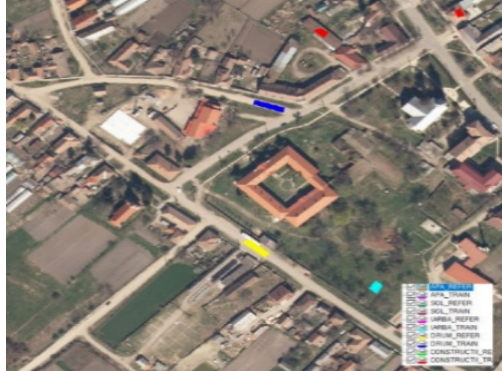

Figure 10. Example of samples digitized for classification

After training the classifiers on the training dataset, the algorithm was applied to the input data; the results of the classification using SVM and MLC are displayed in Fig. 11 and Fig 12.

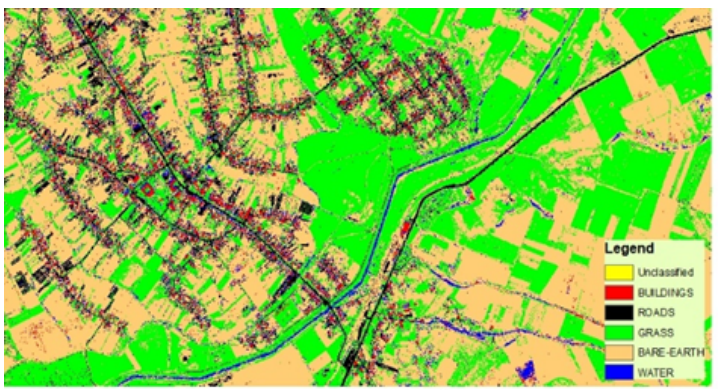

Classes using SVM (\%)

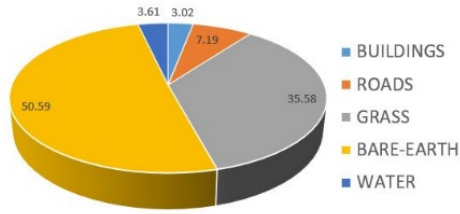

Figure 11. SVM classification result

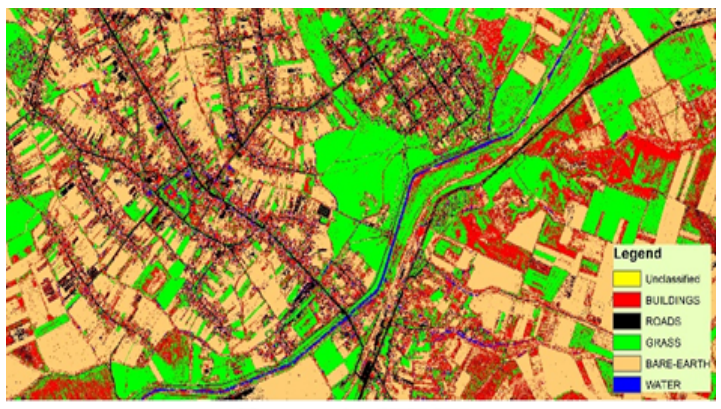

Classes using MLC (\%)

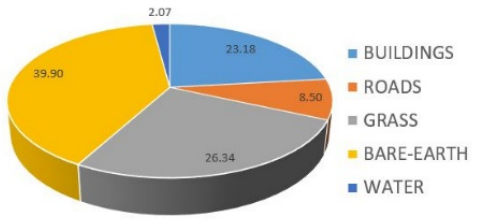

Figure 12. MLC classification result

The percentages for pixels belonging to different classes differ greatly for the class BUILDINGS. When using the SVM classifier the pixels that are classified as BUILDINGS represent $3.61 \%$ from the total amount of pixels, but when applying the MLC classifier the BUILDINGS percentage increases to $23.18 \%$. From a visual analysis, the SVM classifier seems to offer better results in the classification process.

A detailed view of the classification on the city of Săcueni is shown in Fig. 13, where the water bodies are displayed with blue colour and the roads are shown in black. From a visual qualitative inspection, these two classes seem well extracted from the image; after applying successive aggregations, noisy pixels can be removed.

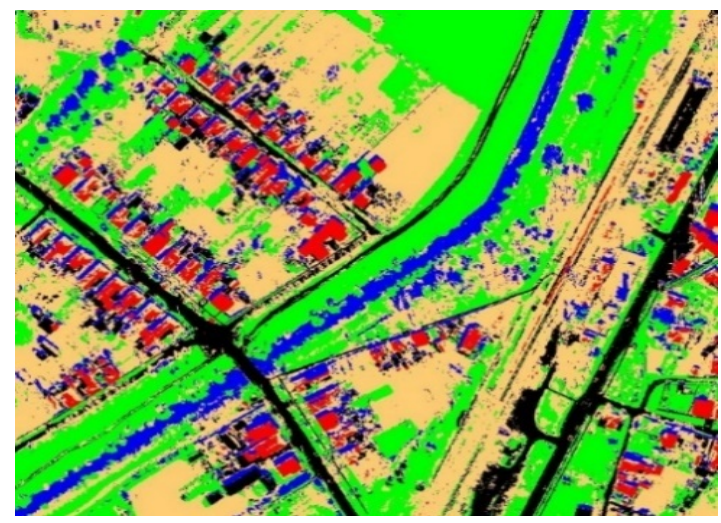

Figure 13. A detailed view of the results with SVM classifier

The final step in the classification process was the accuracy assessment based on the reference dataset previously classified. For the SVM classifier, the overall accuracy is $97.6 \%$ and the Kappa coefficient is 0.97 . The corresponding confusion matrix is displayed in Table 2. The classes ROADS and BAREEARTH generate confusion in the classification process, which is probably due to the fact that the classification is performed only using the RGB bands. For the MLC classifier, the overall accuracy is $97.2 \%$ and the Kappa coefficient is 0.96 , so slightly lower than the SVM result.

\begin{tabular}{|c|c|c|c|c|c|}
\hline Ref. & Buildings & Roads & Grass & $\begin{array}{l}\text { Bare } \\
\text { Earth }\end{array}$ & Water \\
\hline Train. & & & & & \\
\hline Buildings & 10769 & 0 & 0 & 400 & 0 \\
\hline Roads & 0 & $\mathbf{1 8 0 0 3}$ & 1 & $\mathbf{2 7 3 7}$ & 0 \\
\hline Grass & 0 & 0 & 42927 & 0 & 1 \\
\hline Bare Earth & 196 & 104 & 12 & 56942 & 61 \\
\hline Water & 32 & 0 & 0 & 0 & 13533 \\
\hline
\end{tabular}

Table 2. Confusion matrix, SVM classifier

From the accuracy indicators summarized in Fig. 14, it can be concluded that the GRASS class has been well classified. The producer and user accuracies are comparable for all classes using the two classifiers, except for the BUILDINGS class where significant differences are present. 


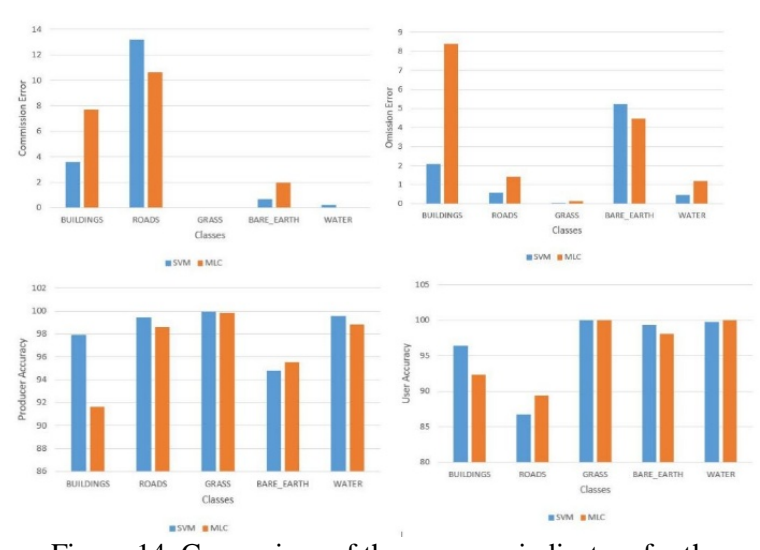

Figure 14. Comparison of the accuracy indicators for the classification of the orthophoto of Săcueni city

To test if more classes would improve the results of the classification, three classes were added to the previous ones, two for different types of BARE-EARTH and one for TREES. Table 3 summarizes the number of samples for each class for both datasets (training and reference).

\begin{tabular}{|c|c|c|c|c|}
\hline \multirow{2}{*}{ Classes } & \multicolumn{2}{|c|}{ Training dataset } & \multicolumn{2}{c|}{ Reference dataset } \\
\cline { 2 - 5 } & $\begin{array}{c}\text { No. } \\
\text { polygons }\end{array}$ & $\begin{array}{c}\text { No. } \\
\text { pixels }\end{array}$ & $\begin{array}{c}\text { No. } \\
\text { polygons }\end{array}$ & $\begin{array}{c}\text { No. } \\
\text { pixels }\end{array}$ \\
\hline BUILDINGS & 35 & 16086 & 35 & 15545 \\
\hline ROADS & 30 & 102646 & 30 & 110799 \\
\hline GRASS & 30 & 276212 & 30 & 276584 \\
\hline BARE-EARTH 1 & 36 & 23555 & 36 & 259188 \\
\hline BARE-EARTH 2 & 23 & 162953 & 23 & 151923 \\
\hline BARE-EARTH 3 & 9 & 36681 & 9 & 39243 \\
\hline TREES & 13 & 54649 & 13 & 49924 \\
\hline WATER & 20 & 9407 & 20 & 84348 \\
\hline
\end{tabular}

Table 3. Number of samples for training and reference dataset, when using 8 classes

The classification algorithm chosen for this approach was MLC (Fig. 15) that is expected to perform better for a larger number of classes. The result for the overall accuracy is $76.1 \%$ and the Kappa coefficient is 0.69 .

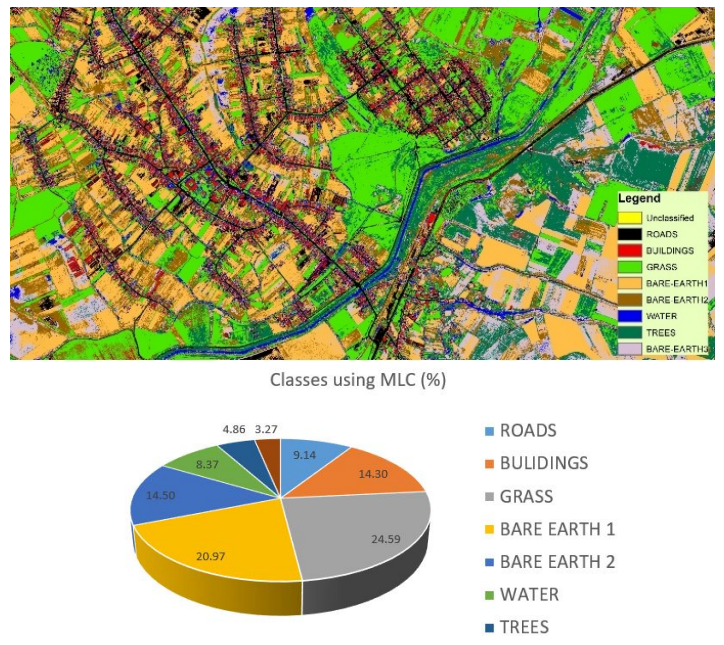

Figure 15. MLC classification with 8 classes
From the indicators in Table 4, the minimum errors are obtained for the GRASS class and the maximum errors occur for the BARE-EARTH 3 class. So, we can concluded that this approach didn't improve the previous classification results.

\begin{tabular}{|c|c|c|c|c|}
\hline \multirow{2}{*}{$\begin{array}{c}\text { Class / } \\
\text { Accuracy } \\
\text { Indicator }\end{array}$} & $\begin{array}{c}\text { Comm. } \\
\text { Error }\end{array}$ & $\begin{array}{c}\text { Omis. } \\
\text { Error }\end{array}$ & $\begin{array}{c}\text { Producer } \\
\text { Accuracy }\end{array}$ & $\begin{array}{c}\text { User } \\
\text { Accuracy }\end{array}$ \\
\cline { 2 - 5 } & \multicolumn{2}{|c|}{ Percent } & \multicolumn{2}{c|}{ Percent } \\
\hline ROADS & 14.88 & 6.48 & 93.52 & 85.12 \\
\hline BUILDINGS & 9.55 & 36.72 & 63.28 & 90.45 \\
\hline GRASS & $\mathbf{2 . 3 5}$ & $\mathbf{2 . 9 1}$ & $\mathbf{9 7 . 0 9}$ & $\mathbf{9 7 . 6 5}$ \\
\hline BARE-EARTH 1 & 22.42 & 25.27 & 74.73 & 77.58 \\
\hline BARE-EARTH 2 & 40.75 & 39.37 & 60.63 & 59.25 \\
\hline WATER & 21.68 & 5.03 & 94.97 & 78.32 \\
\hline TRESS & 46.14 & 52.33 & 47.67 & 53.86 \\
\hline BARE-EARTH 3 & 85.9 & 75.46 & 24.54 & 14.1 \\
\hline
\end{tabular}

Table 4. Accuracy assessment using the MLC approach for the dataset with 8 classes (the best results are highlighted)

\subsection{Classification of Sentinel Images}

The second image dataset on the city of Săcueni tested for classification was acquired by Sentinel-2A MSI sensor (Fig. 16) on 30.08.2019. The data was downloaded from https://scihub. copernicus.eu/.

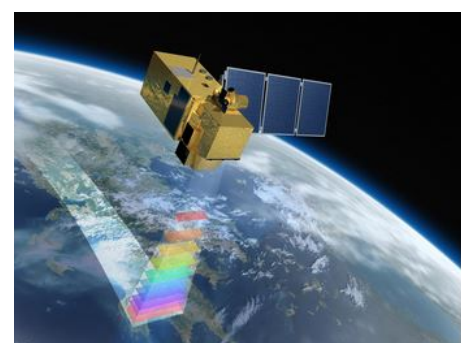

Figure 16. Sentinel-2 ("Sentinel 2, a valuable tool in environmental studies", 2017)

The Copernicus Sentinel-2 mission comprises a constellation of two polar-orbiting satellites placed in the same sun-synchronous orbit, phased at $180^{\circ}$ to each other.

It aims at monitoring Earth's surface changes and has a wide swath width $(290 \mathrm{~km})$ and high revisit time (5/10 days). It carries an optical instrument payload that samples 13 spectral bands, with the following spatial resolutions: 10 meters (4 bands), 20 meters ( 6 bands) and 60 meters ( 3 bands). Sentinel- 2 products available for users are at two different levels: $1 \mathrm{C}$ and 2A (https://sentinel.esa.int). Level-1C products provide the top of atmosphere reflectances in fixed cartographic geometry, so the radiometric and geometric corrections are applied. Level-2A products provide the bottom of atmosphere reflectance in a geospatial reference system. This product is atmospherically corrected, based on the libRadtran radiative transfer model (Mayer, 2005), using the Sen2Cor processor and PlanetDEM Digital Elevation Model. In this work images with Level 2A were used.

The downloaded files contain 13 different images, one for each spectral band. For classification the four bands in the visible and near-infrared spectrum with ground resolution of 10 meters were used (Fig. 17). 


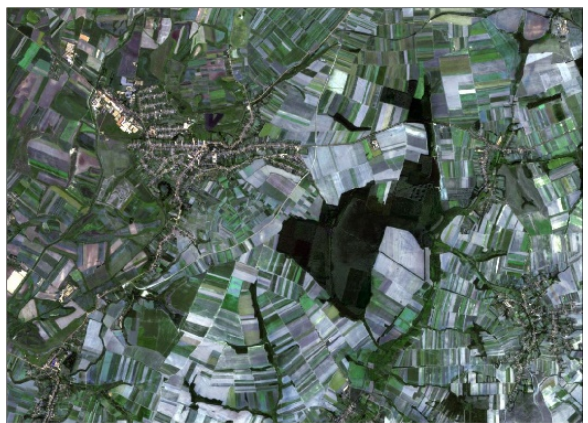

Figure 17. Satellite imagery over the city Săcueni

In the process of image interpretation, five classes were defined: urban area, forest, arable land, bare earth and roads; as for the airborne case, SVM and MLC classifiers were applied. For the training dataset, 70 samples were used, the same as for the reference set (Fig. 18).

\begin{tabular}{|c|c|c|c|c|}
\hline \multirow{2}{*}{ CLASSES } & \multicolumn{2}{|c|}{ TRAINING SET } & \multicolumn{2}{c|}{ REFERENCE SET } \\
\cline { 2 - 5 } & POLYGONS & PIXEL & POLYGONS & PIXEL \\
\hline ARABLE LAND & 15 & 1891 & 15 & 2034 \\
\hline BARE EARTH & 15 & 2405 & 15 & 2516 \\
\hline ROADS & 15 & 354 & 15 & 542 \\
\hline URBAN AREAS & 13 & 1401 & 13 & 1101 \\
\hline FOREST & 12 & 1137 & 12 & 1147 \\
\hline
\end{tabular}

Figure 18. Statistics samples for the satellite image

The application of the SVM and MLC classifiers following the training process led to the results presented in Fig. 19 and Fig 20. The results are showing that for ROADS class, the MLC ranks more pixels than SVM, but after the visual inspection we can state that not all ROADS pixels are well classified.

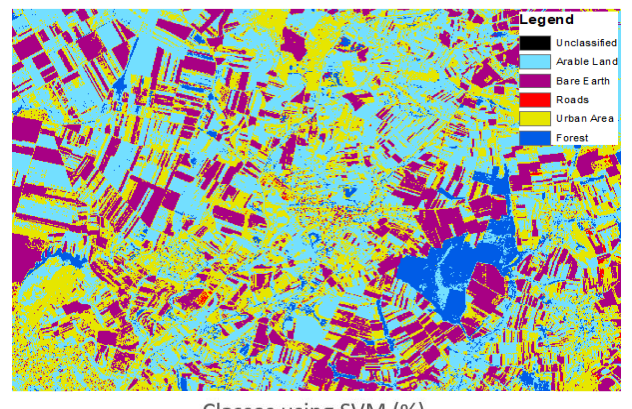

Classes using SVM (\%)

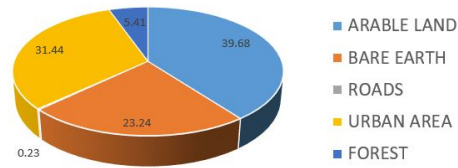

Figure 19. SVM classification of Sentinel-2 image
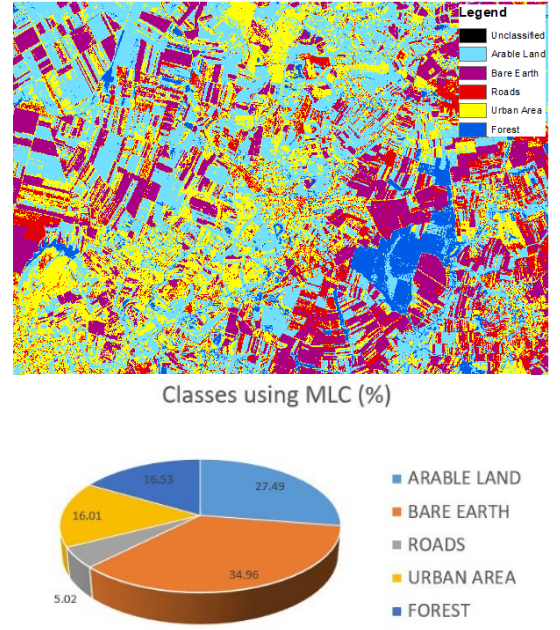

Figure 20. MLC classification of Sentinel-2 image

For the SVM classifier, the pixels classified as URBAN AREAS and FOREST increased inversely proportionally, if compared to the MLC classifier.

The results of the classification process for the city of Săcueni are visualized in Fig. 21. By a visual check, it can be confirmed that the pixels classified in the ROADS class with SVM are more than those classified with MLC.

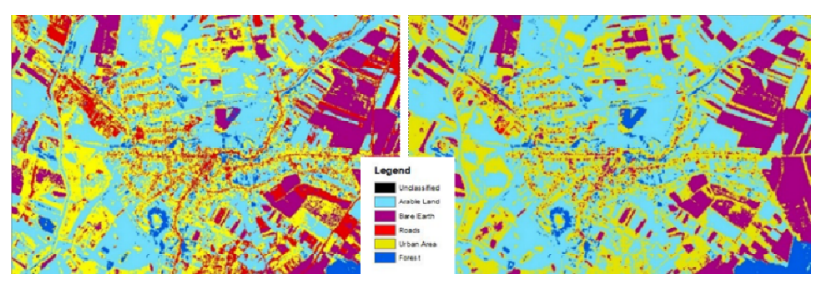

Figure 21. Comparison between the results obtained from MLC and SVM classification algorithms

The accuracy assessment executed in the post-classification process with the reference dataset, produced the confusion matrix and the indicators, showing that the indistinctness between the classes that led to the result of the classification. The accuracy indicators are computed for each class and summarized in Fig. 22. The ROADS class has the worst results in the classification process. The producer and user accuracies are comparable for most of the classes for both classifiers, except for the ROADS class where bigger differences are evident.

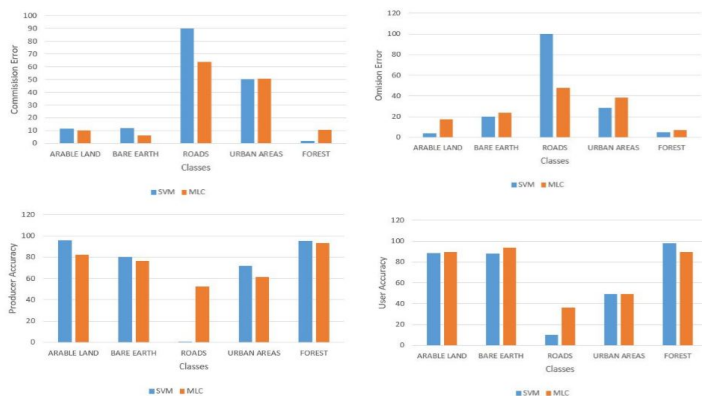

Figure 22. Comparison of the accuracy indicators for the satellite image of Săcueni city 
Based on the results shown, it was decided to eliminate the class ROADS from the classification process. The image was reinterpreted and configured on four classes (URBAN AREAS, FOREST, ARABLE LAND and BARE EARTH). From the available 144 samples, $40 \%$ were used in the training dataset and $60 \%$ in the reference dataset.

The results obtained from the new training dataset on the same satellite image, and applying the same two algorithms, are shown in Fig. 23 and Fig. 24. The approach using MLC gives an overestimated result for URBAN AREAS class, with a growth of about $11 \%$ if compared with the approach using SVM (Fig. 24).

The accuracy indicators calculated using the reference dataset show that by reducing the number of classes the results are more precise, both visually and numerically.

Also, in the case of satellite images, based on the accuracy assessment summarized in Fig. 25, SVM classifier performs a better classification than MLC classifier.

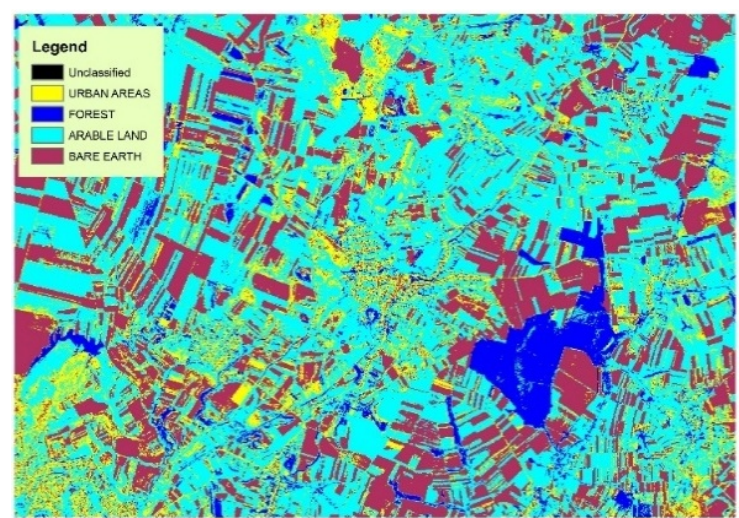

Classes using SVM (\%)

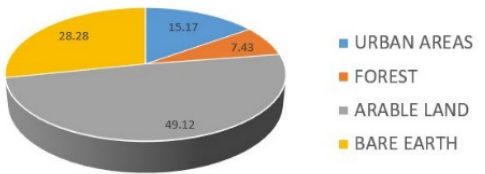

Figure 23. SVM classification result for the satellite image, when using 4 classes

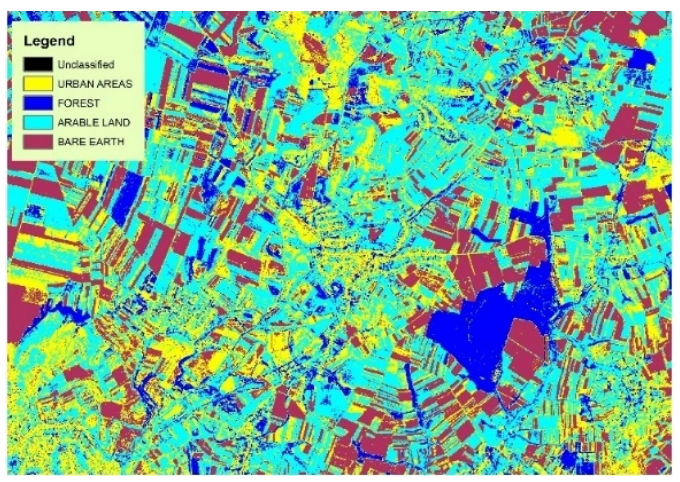

Classes using MLC (\%)

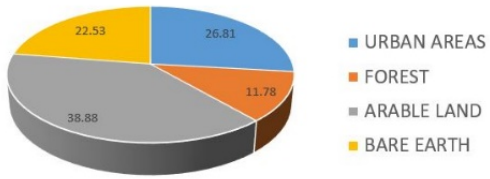

Figure 24. MCL classification result for the satellite image, when using 4 classes

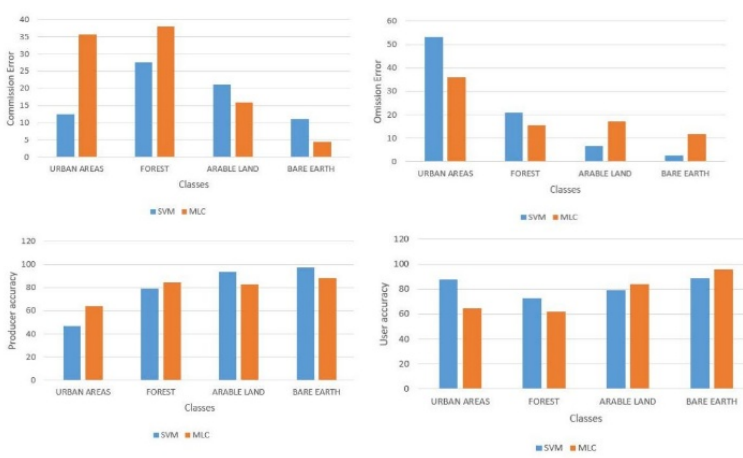

Figure 25. Accuracy assessment for classification of the satellite image over Săcueni city (4 classes)

The final classification result of the SVM classifier on Sentinel2A image on Săcueni city is shown in Fig. 26. After a visual inspection, it can be concluded that these classes are properly extracted.

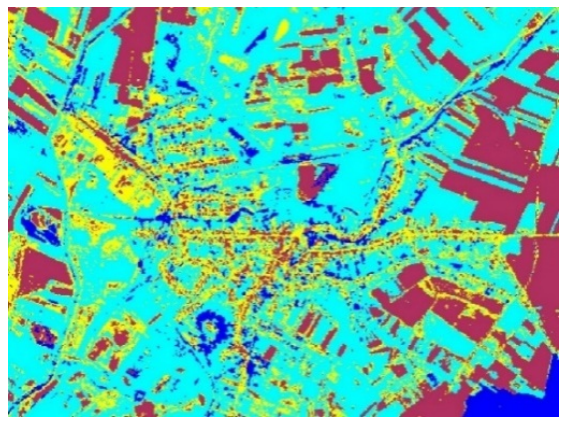

Figure 26. Results obtained for SVM classification algorithms, for Săcueni city

\section{CONCLUSIONS}

In this paper it is shown that by using more classes and samples on the aerial orthophoto, the result of the classification does not improve, but more confusion between classes appear and the likelihood of an object being in the corresponding class is reduced. For the SVM classifier, a smaller number of classes and samples gives the best results for the classification. Subsequently, the results can be post-processed to obtain an image with higher accuracy per class and less noise in the data. Therefore it can be concluded that the SVM classifier, for both images used in the case study, offers higher accuracy in the classification process. For extra-urban areas or large areas it is 
most likely to use satellite images, but for urban areas or small areas, the aerial images with appropriate spatial resolution are the best choice. The results of classification depend on the distribution and completeness of the datasets used for training and as reference and on the number of classes chosen.

The confusion matrix can offer valuable information about the classes with similar features, so the classes that create confusion can be eliminated from the process, or the corresponding samples can be edited (if possible). The processing time in ENVI software is less for the MLC classifier than for SVM one. To extract objects from the classified images the workflow has to be extended; in fact the two classifiers are pixel-based and the accuracy indicators refer to the pixel, not to the object.

\section{ACKNOWLEDGEMENTS}

The authors thank the National Centre of Cartography and the National Agency of Cadastre and Land Registration for providing the aerial imagery, the colleagues from the University of Warsaw, partners in the VOLTA project (Grant Agreement no. 734687 - VOLTA - H2020-MSCA-RISE-2016) who provided with the ENVI licenses used for the classification process. Finally, the authors acknowledge ESA for the Sentinel dataset.

\section{References:}

Foody, M. G., 2002. Status of Land Cover Classification Accuracy Assessment, Remote Sensing of Environment, 80, pp. 185-201.

Foody, M. G., Mathur, A., 2004. A Relative Evaluation of Multiclass Image Classification by Support Vector Machines. IEEE Transactions on Geoscience and Remote Sensing, 42, pp. 1335 1343.

Karbo, N., Schroth, R., 2009. Oblique aerial photography: a status review. Proc. 52nd Photogrammetric Week, pp. 119-125.

Kamavisdar, P., Saluja, S., Agrawal, S., 2013. A Survey on Image Classification Approaches and Techniques. International Journal of Advanced Research in Computer and Communication Engineering, $2(1)$.

Leberl, F., Thurgood, J., 2004. The Promise of Softcopy Photogrammetry Revisited. Theme Session 12: Automated Object Extraction and Computer Vision Application .

Lemmens, M., 2011. Digital aerial cameras. GIM International, 25(4).

Mayer, B., Kylling, A., 2005. Technical note: The libRadtran software package for radiative transfer calculations- description and examples of use, Atmos. Chem. Phys., 5, pp. 1855-1877, doi: 10.5194/acp-5-1855.

Navalgund, R. R., 2001. Remote sensing. Reson, 6, pp. 51-60, https://doi-org.am.e-nformation.ro/10.1007/BF02913767.

Petrie, G., 2009. Systematic aerial oblique photography using multiple digital cameras. Photogrammetric Engineering \& Remote Sensing, pp. 102-107.

Remondino, F., Rupnik, E., Nex, F., 2014. Oblique Multi-Camera Systems - Orientation and Dense Matching Issues. ISPRS, XL3/W1.
Wolf, P. R., Dewitt, B. A., Wilkinson, B. E., 2014. Elements of Photogrammetry with Application in GIS. McGraw-Hill Education, 4th Edition, New York.

Vreys, K., Iordache, M. D., Bomans, B., Meuleman, K., 2016. Data acquisition with the APEX hyperspectral sensor. Miscellanea geographica - regional studies on development, 20 (1), pp. 5-10, https://doi.org/10.1515/mgrsd-2016-0001.

Duda, R., Hart, P., 1973. Pattern classification and scene analysis. A Wiley-Interscience publication.

Linder, W., 2006. Digital Photogrammetry A Practical Course. Springer, New York.

Kramer, J. H., 2002. Observation of the earth and its environment: Survey of missions and sensors, 4th Edition.

Schenk, T., 2005. Introduction to Photogrammetry. Department of Civil and Environmental Engineering and Geodetic Science, Ohio State University, pp. 3-4.

Vapnik, V. N., 1995. The Nature of Statistical Learning Theory. Information Science and Statistics. Springer-Verlag.

"HySpex", https://www.hyspex.com (view at 10 August 2020).

"DigitalGlobe", http://worldview3.digitalglobe.com (view at 11 August 2020).

"Boston as the eagle and the wild goose see it", http://bit.ly/2m6wgXa (view at 30 August 2020).

"Aerial Photography and Remote Sensing", last modified September 11, 2014, https://web.archive.org/web/20141030095245/http://www.colorado. edu/geography/gcraft/notes/remote/remote_f.html.

"First photo from space", last edited March 27, 2020, https://commons.wikimedia.org/wiki/File:First_photo_from_space.j pg.

"Landsat Program", https://landsat.gsfc.nasa.gov (view at 03 September 2020)

"Sentinel 2", Copyright () 2012 European Space Agency, https://sentinel.esa.int/documents/247904/349490/S2_SP_ 1322_2.pdf.

Gandhi, R., "Machine learning algorithms", last modified June 7, 2018, https://towardsdatascience.com/support-vector-machineintroduction-to-machine-learning-algorithms-934a444fca47.

"Sentinel", https://sentinel.esa.int (view at 03 September 2020).

"Sentinel 2, a valuable tool in environmental studies", last modified March 4, 2017, https://www.cls.fr/en/sentinel-2-environmentalstudies. 